\title{
Epicardial adiposopathy and atrial fibrillation
}

Submitted: 20 October 2015; Accepted: 27 October 2015; Published online: 04 November 2015

Atrial fibrillation (AF) is the most frequent heart rhythm disorder in clinical practice. Many conditions could predispose the development of AF such as hypertension, heart failure, valvular heart disease, aging, coronary artery disease, diabetes mellitus, and sleep apnoea [1-6].

Kocyigit and colleagues reported a study to investigate the association between epicardial adipose tissue (EAT) thickness and $\mathrm{AF}$ recurrence after cryoballoon-based pulmonary vein isolation (PVI). A total of 249 patients $(55.6 \pm 10.7$ years; $48.2 \%$ male; $18.5 \%$ persistent AF were followedup for 29 months ( 8 months- 48 months). AF after the ablation procedure was $75.9 \%$ at a median follow-up of 29 months.

Total periatrial EAT thickness $(18.1 \pm 6.2$ vs. $14.7 \pm 4.7 \mathrm{~mm} ; \mathrm{P}<0.001)$ was greater in patients with late $\mathrm{AF}$ recurrence when compared to those without. Periventricular or total EAT thickness measurements did not differ between both groups $(\mathrm{P}>0.05)$. Multivariate Cox proportional hazard regression analysis showed that periatrial EAT thickness (hazard ratio, 1.086; $\mathrm{P}=0.001$ ) and left atrial volume index (hazard ratio, 1.144; $\mathrm{P}<0.001$ ) were independent predictors for late AF recurrence. EAT thickness may serve as a beneficial parameter for prediction of AF recurrence after cryoballoon- based PVI [7].
Venteclef and colleagues examine the effects of the secretome of human EAT on the histological properties of the myocardium. Samples of EAT and subcutaneous adipose (SAT), obtained from 39 patients undergoing coronary bypass surgery, were analysed and tested in an organo-culture model of rat atria to evaluate the fibrotic properties of human fat depots.

The EAT secretome induced global fibrosis (interstitial and peripheral) of rat atria in organo-culture conditions. Activin A was highly expressed in EAT compared with SAT and promoted atrial fibrosis, an effect blocked using neutralizing antibody. Active in A levels were enhanced in patients with low leftventricular function. In sections of human atrial and ventricular myocardium, adipose and myocardial tissues were in close contact, together with fibrosis.

The secretome from EAT promotes myocardial fibrosis through the secretion of adipo-fibrokines such as Activin A [8]. EF is associated with AF. A large cohort study should be conducted to definitively determine the clinical significance of EF, its correlation with AF. Potential treatments such as lifestyle modification, ectopic fat reduction, and medications should be investigated.
Leonardo Roever* \&

Elmiro Santos Resende Department of Clinical Research, Federal University of Uberlandia, Brazil *Author for correspondence: Tel.: 55348803987

leonardoroever@hotmail.com 


\section{References}

1. Benjamin EJ, Wolf PA, D'Agostino RB, et al. Impact of atrial fibrillation on the risk of death: the Framingham Heart Study. Circulation. 98, 946-952 (1998).

2. Hatem SN, Sanders P. Epicardial adipose tissue and atrial fibrillation.Cardiovascular Research 102, 205-213 (2014).

3. Benjamin EJ, Levy D, Vaziri SM, et al. Independent risk factors for atrial fibrillation in a population-based cohort. The Framingham Heart Study. JAMA 271, 840-844 (1994).

4. Sanders P, Morton JB, Kistler PM, et al. (2004) Electrophysiological and electroanatomic characterization of the atria in sinus node disease: evidence of diffuse atrial remodeling. Circulation. 109: 1514-1522.

5. Ohn B, Stiles MK, Kuklik P, et al. Electrical remodelling of the left and right atria due to rheumatic mitral stenosis. Eur Heart. J. 29, 2234-2243 (2008).

6. Ohn B, Stiles MK, Kuklik P, et al. Reverse remodeling of the atria after treatment of chronic stretch in humans: implications for the atrial fibrillation substrate. J. Am. Coll. Cardiol. 55, 1217-1226 (2010).

7. Kocyigit D, Gurses KM, Yalcin MU, et al. Periatrial epicardial adipose tissue thickness is an independent predictor of atrial fibrillation recurrence after cryoballoon-based pulmonary vein isolation. J. Cardiovasc. Comput. Tomogr. 9, 295-302 (2015).

8. Venteclef N, Guglielmi V, Balse E, et al. Human epicardial adipose tissue induces fibrosis of the atrial myocardium through the secretion of adipo-fibrokines. Eur. Heart. J. 36: 795-805 (2015). 